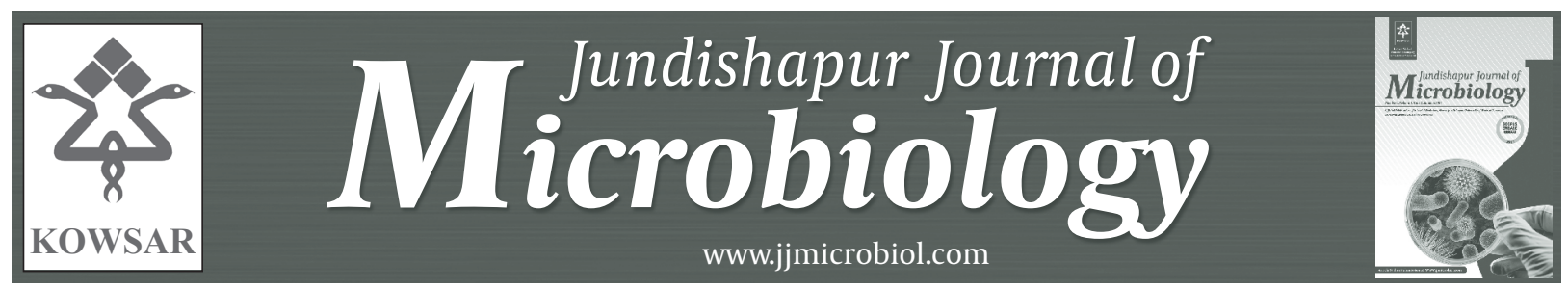

\title{
Effect of Silver Nanoparticles on Common Bacteria in Hospital Surfaces
}

\author{
Fatemeh Ahmadi ${ }^{1}$, Sara Abolghasemi ${ }^{2}$, Najme Parhizgari ${ }^{2}$, Fatemeh Moradpour ${ }^{2}$ \\ ${ }^{1}$ Infectious and Tropical Diseases Research Center, Ahvaz Jundishapur University of Medical Sciences, Ahvaz, IR Iran \\ ${ }^{2}$ Ahvaz Jundishapur University of Medical Department Sciences, Ahvaz, IR Iran \\ ${ }^{*}$ Corresponding author: Sara Abolghasemi, Ahvaz Jundishapur University of Medical Department Sciences, Ahvaz, IR Iran, Tel.: +98-2155391100, E-mail: sara- \\ habolghasemi@dr.com.
}

\section{A B S T R A C T}

Background: One of the most important causes of complications and mortality in medical centers are nosocomial infections. Disinfection of hospital surfaces is essential element for ensuring that infectious agents are not transmitted to patients. Alcoholbased and chlorine-based disinfectants have unfavorable properties. Given that the antimicrobial effect of heavy metals such as silver is recognized as a viable option for eliminating bacteria, the exploration of nanotechnology in this context has been described in this study. Nanotechnology uses both science and technology to produce new materials with nano-scales.

Objectives: The effect of silver nanoparticles on some common hospital bacteria has been studied in this research.

Patients and Methods: We have selected nine patients' metal file covers and following sterilizing, we have infected them with one of these bacteria; Staphylococcus aureus, Pseudomonas aeruginosa and Bacillus cereus. Then, the infected surfaces have been disinfected with different dilutions of silver nanoparticles. Sampling and culturing has done following four specific intervals. Afterwards, the colonies that developed have been counted and compared.

Results: All of the three dilutions of silver nanoparticles could bring the colony count out of 7.5x106 to less than 100 which indicate more than a 99 percent reduction. No remarkable difference of the three dilutions of disinfectants was observed in reducing the colony count in 5,15,30 and 60 minute disinfection intervals (P value $>0.05$ ). The effect of constant dilution of silver nanoparticles on the reduction of organisms varied in response to the time of disinfection (P value $<0.05)$.

Conclusion: Silver nanoparticles had appropriate effects in all three types of dilutions and allowing for a more protracted contact time has given significantly better results.

Keywords: Disinfection; Nosocomial Infection; Disinfectant.

Copyright @ $\odot 2013$, Ahvaz Jundishapur University of Medical Sciences; Published by Kowsar Corp.

\footnotetext{
-Article type: Research Article; Received:26 Feb 2012; Revised:22 May 2012; Accepted:20 Jun 2012 ; DOI: 10.5812/jjm.4585

-Implication for health policy/practice/research/medical edjucation: It seems that silver nanoparticles have a useful antimicrobial effect, and could be utilized as a viable alternative disinfectant in hospitals as described in this manuscript.

-Please cite this paper as:

Ahmadi F, Abolghasemi S, Parhizgari N, Moradpour F. Effect of Silver Nanoparticles on Common Bacteria in Hospital Surfaces. Jundishapur J Mirobiol. 2013; 6(3): 209-14. DOI: 10.5812/jjm.4585
}

Copyright @ 2013, Ahvaz Jundishapur University of Medical Sciences; Published by Kowsar Corp.

This is an Open Access article distributed under the terms of the Creative Commons Attribution License (http://creativecommons.org/licenses/by/3.0), which permits unrestricted use, distribution, and reproduction in any medium, provided the original work is properly cited. 


\section{Background}

Nosocomial infections are one of the most important causes of complications and mortality in medical centers. These infections have been considered as the sixth leading cause of death in US and Europe. As well, they contribute 10-50 billion dollars to the economic burden annually (1). On the other hand, devices used for patient care were divided in to three groups based ontheriskofbeing infectious; critical items, semi-critical items and non-critical items.

Critical items enter sterile tissue and the vascular system. Therefore, if they are contaminated with microorganisms such as bacterial spores there is a high risk of infection. Therefore these items should be sterile (2).

Semi-critical items have contact with mucous membranes and skin that has been compromised. So, there is a necessity to disinfect microorganisms with a highlevel chemical disinfectant $(2,3)$. Non-critical items have contacts with skin, but not with mucous membranes. They are divided in to two groups: non-critical patient care items and non-critical environmental surfaces (2, 3).

Examples for non-critical patient care items are the bedpan, blood pressure cuff and computer system. In fact, they are not dangerous and do not infect patients with infectious agents (4). Non-critical surfaces include: the patients' bed rail, the table beside patients' bed, that have repeated contact with hands and can potentially contribute to transmitting secondary infectious agents by infecting hospital staffs' hands and other medical items which have contact with the patients $(5,6)$.

Disinfection is a process that eradicates some or the entire pathogen microorganisms except bacterial spores on inanimate surfaces (7). Disinfectants threaten healthcare workers' healthfrom mucosal irritation to death (8).However, toxicity degrees are different $(9,10)$ but all disinfectants must be used with precautions (11). Based on the efficiency grade, disinfectants are divided in to three levels: high, medium and low (12).

Comprehensive studies on anti-septic (13) and disinfectants (14) clearly indicate the anti-microbial effect of heavy metals $(15,16)$. Silver was used for prevention of newborns' conjunctivitis, as a topical treatment for burn trauma and impregnating a different catheter (17).

Nano-technology is a new developing context using science and technology with the aim of producing new materials with nano-scales (18). The term nano-technology was first created by Professor Nario Taniguchi in Japan in 1934 that described producing precise materials in nano-scales (19). Different types of nano materials include titanium (20), gold (21), alginate (22) and silver have been studied however the antimicrobial effect of silver nanoparticles demonstrated that it is the most effective substance against bacteria, viruses and eukaryotic microorganisms (23). This superior result is because of the very large surface that causes better contact with microorganisms. Nanoparticles adhere to the cell membrane and permeate in to the bacterium (24-26).

Using silver nanoparticles as a disinfectant may provide concerns about the risk of silver toxication (Argyria) and its toxicity for the body cells (27). Research in this field indicate that silver ions or nano-particles can be applied for the treatment of burns, dental materials, sunscreen lotions and so on. It has low toxicity for human cells (24).

\section{Objectives}

Alcohol-based and chlorine-based disinfectants have adverse side effects such as irritations, skin allergies and inhalation risks as well as an annoying and offensive odor. The disinfection effect of these substances on the multitude of existing resistant microorganisms is controversial. For this reason, we studied the effect of the different dilutions of silver nanoparticles on some of the common hospital organisms at specific intervals. Provided that the effect of silver nanoparticles proves to be an effective, viable alternative to traditional hospital disinfectants, we recommend that this substance be used in their stead.

\section{Patients and Methods}

In a single blind interventional study, we have selected nine patients' metal file covers as representative of common hospital surfaces. We sterilized these surfaces with an autoclave before beginning the intervention. Subsequently, we collected swab samples from those surfaces and cultured them to ensure that we have reached the objective of sterile surfaces. If there is any culture growth on these surfaces, that surface will be excluded from the study and the test as described will be repeated.

\subsection{Contaminating Surfaces}

We have dedicated four equal $2 \times 2 \mathrm{~cm}$ squares on every sterile metal cover (a, b, c, d). Sterile surfaces were infected with 50microliters of the selected organisms under study with concentration of 0.5 McFarland. Regarding that 1 milliliter of 0.5 McFarland contains $1.5 \times 10^{8}$ bacteria, we have used $7.5 \times 10^{6}$ bacteria to contaminate every $2 \times 2 \mathrm{~cm}$ surface. Bacteria were then extracted from the positive clinical samples of the hospital laboratory and their strains have been verified by standard biochemical tests. We have dedicated 3 file cover surfaces for each kind of bacteria with the aforementioned four $2 \times 2 \mathrm{~cm}$ squares on each surface. 
The culture media contained the following kinds of bacteria colonies: methicillin resistant Staphylococcus aureus MRSA (as a common nosocomial infection and colonization Gram-positive bacteria), Pseudomonas aeruginosa (with antibiogram resistant to ceftazidime and cefepime as common hospital Gram-negative bacteria) and Bacillus cereus (as common nosocomial infection and colonization sporulative bacteria). A 0.5 McFarland suspension of each culture media was prepared and every four squares (a, b, c, d) of file covers have been contaminated with the amount of bacteria (50 microliter) as follows: files 1-2-3 (s1, s2, s3) contaminated with $S$. aureus, files 4-5-6 (p1, p2, p3) with $P$. aeruginosa and files 7-89 (b1, b2, b3) with B. cereus.

\subsection{Disinfection of Contaminated Surfaces}

All squares on the nine metal covers have been disinfected witha swab which was saturated with one of the three silver nanoparticles' dilutions (100ppm, 200ppm, $300 \mathrm{ppm}$ ). To break it down, we had four squares on $s 1$, p1, b1 with 100ppm silver nanoparticles, four squares ons2, p2, b2 with 200ppm silver nanoparticles and four squares ons3, p3, b3 with 300ppm silver nanoparticles. It was assumed that PPM means part per million, and silvernano-particles were diluted with sterile water to these dilutions.

\subsection{Sampling and Culturing}

Following 5 minutes, the square (a) of each metal cover had been sampled by a sterile swab and this swab was put in a test tube containing normal saline. Until then, it had been transferred to the culture media. Sampling was repeated for b, c and d squares after 15, 30 and 60 minutes, respectively. The encoded test tubes had been put at a microbiologist's disposal. The microbiologist was unaware of the bacteria type, the disinfectant substance used in each test tube and the duration time given for disinfection of each test tube's swab sample. Hence, this research is a single blind interventional study.

Test tubes was placed in the Vortex, Vibrofix VF1 IKA Germany,(a machine for equalizing suspended solids) immediately following sampling, then 200 microliters of the content of each tube was entered in to a separated Mueller-Hinton agar culture medium (Merck, Germany) in equal and sterile conditions. The colony count from each bacterium was calculated following incubation in 37 degrees centigrade for 24 hours. Study has compared the effect of different dilutions of silver nanoparticles on colony count reduction in a constant time as well as comparing the effect of each dilution of this substance on colony count reduction in different disinfection times in the same environmental conditions.

All the experiments were repeated two other times on the same metal covers and prior each time the studying surfaces had been sterilized again for ensuring the accuracy of the study. We have used the provided forms for recording observations and announced laboratory results as data collection method and tools and have calculated the average bacteria colony count of three time interlude experiments. Each colony count is calculated after a specific disinfection time. Statistical analysis had carried out using analysis of variance and repeated measures tests. $P$ value $<0.05$ was considered meaningful.

\section{Results}

No microorganisms grew in the first samples collected from sterile surfaces. Therefore no surface excluded from the study. We calculated the average colony count divided by the type of microorganism and the total average of counted bacteria for each specific time period divided by the disinfectant dilution. The results can be seen as recorded in Table 1 .

All the three dilutions of silver nanoparticles had definite positive effects on the bacteria under study as can be seen in Table 1 . The silver nanoparticles could bring the colony count out of $7.5 \times 10^{6}$ to less than 100 which indicates more than 99 percent reduction. No remarkable difference was indicated in comparing different dilutions of silver nanoparticles on the total of all microorganisms which were applied for contaminating surfaces in a specific time $(P$ value $>0.05$ in all four specific times, Figure 1 ).

Table1. Mean Number of Calculated Studying Bacteria Colony Count in the Culture Media Following Times of Disinfection

\begin{tabular}{lllllllllllll}
\hline Disinfectant & \multicolumn{4}{l}{ Silver Nanoparticles $\mathbf{1 0 0} \mathbf{~ p p m}$} & \multicolumn{3}{l}{ Silver Nanoparticles 200 ppm } & \multicolumn{4}{c}{ Silver Nanoparticles 300 ppm } \\
\hline $\begin{array}{l}\text { Bacteria/ } \\
\text { Time }\end{array}$ & 5 & 15 & 30 & 60 & 5 & 15 & 30 & 60 & 5 & 15 & 30 & 60 \\
MRSA $^{\text {a }}$ & $10 / 33$ & 1 & $0 / 33$ & 0 & $3 / 66$ & 2 & $0 / 33$ & 0 & $13 / 33$ & $0 / 33$ & 0 & 0 \\
P.A $^{\text {a }}$ & 34 & 0 & 0 & 0 & $33 / 66$ & 0 & 0 & 0 & $43 / 33$ & 0 & 0 & 0 \\
B.C $^{\text {a }}$ & $46 / 66$ & $19 / 33$ & 2 & 1 & $67 / 66$ & $35 / 33$ & $1 / 33$ & $0 / 33$ & $34 / 66$ & $0 / 66$ & $0 / 33$ & 0 \\
Mean of T. $^{\text {a }}$ & $62 / 8$ & $6 / 77$ & $0 / 77$ & $0 / 33$ & $34 / 9$ & $12 / 44$ & $0 / 55$ & $0 / 11$ & $26 / 3$ & $0 / 33$ & $0 / 11$ & 0 \\
\hline
\end{tabular}

a Abbreviations: Mean of T, Mean of Total of all Bacteria; MRSA, Methicillin Resistant Staphylococcus; PA, P. aeuroginosa; BC, Bacillus Cereus 


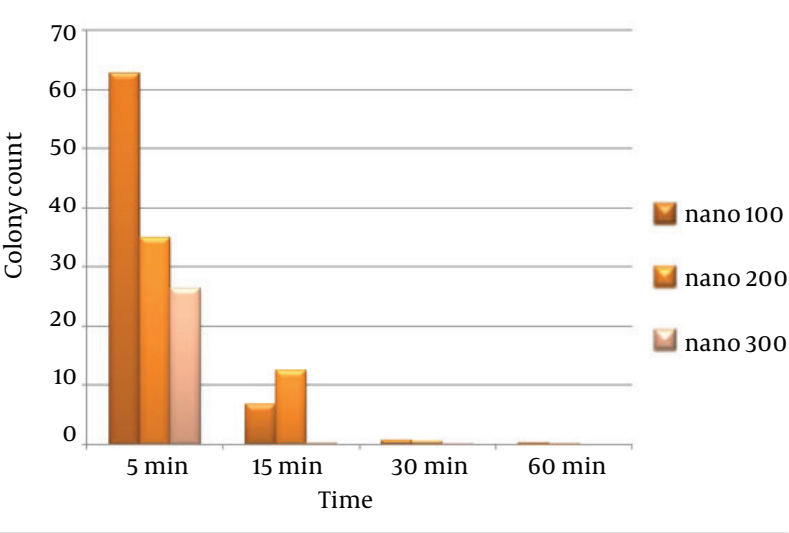

Figure 1. Comparing Different Dilutions of Silver Nanoparticles' Efficacy on Total Bacteria in Different Times

Although 300ppm silver nanoparticles showed a significant efficacy in 15, 30 and 60minutes, the difference in efficacy between intervals had no statistical meaning, as mentioned beforehand. By allowing longer contact time with each dilution of disinfection in our study, we had a significant colony count reduction compared to the former specific contact time (P value $<0.05)$. 100ppm, 200ppm and 300ppm silver nanoparticles had respectively $P$ value of $0.01,0.03$, and 0.01 . The calculated average total number of colony count for each three types of bacteria was calculated comparatively with definite disinfection time in culture media divided by the dilution of the disinfectants as is indicated in the Figures 2, 3,4.

\section{Discussion}

Following five minutes all disinfectants reduced the S. aureus colony count significantly (more than 99\%), but none of them could bring the $S$. aureus colony count to zero. This rate was achieved following 30 minutes of disinfection with 300ppm silver nanoparticles.

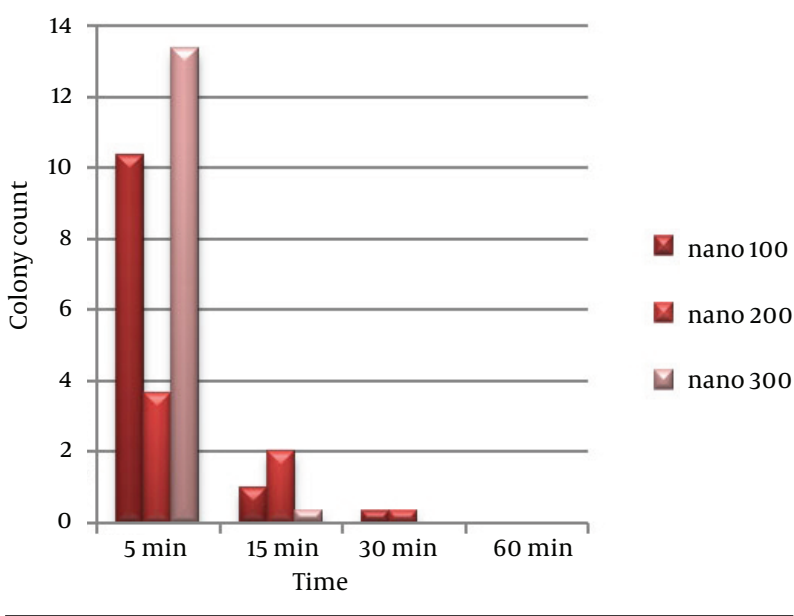

Figure 2. Comparing Different Dilutions of Silver Nanoparticles' Efficacy onS. aureus in Different Times

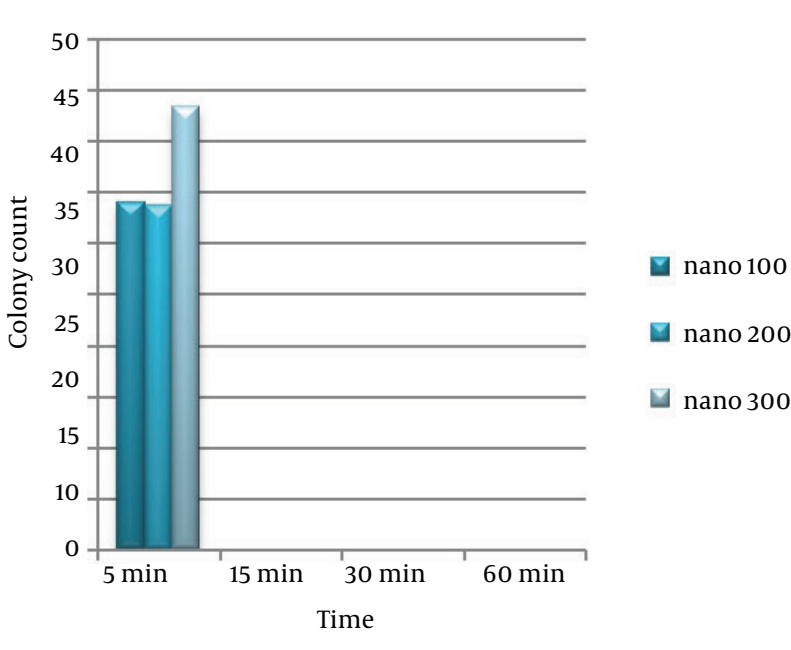

Figure 3. Comparing Different Dilutions of Silver Nanoparticles' Efficacy on P. aeruginosa in Different Times

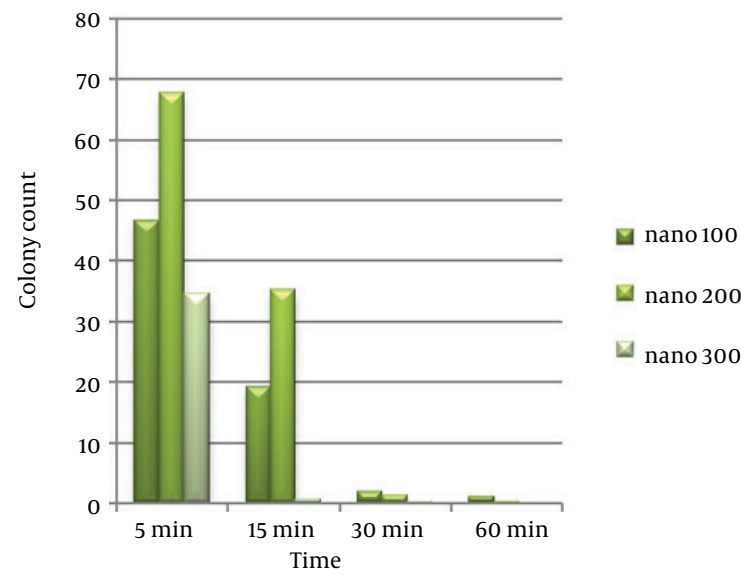

Figure 4. Comparing Different Dilutions of Silver Nanoparticles' Efficacy on Bacillus Cereus in Different Times

For two other dilutions of silver nanoparticles, this rate was possible after 60 minutes of disinfection. Thus 300ppm effect of silver nanoparticles on reducing $S$. aureus colony count is faster than the two other ones, but no remarkable difference of the three dilutions of disinfectants was observed in reducing S. aureus colony count in 5,15, 30 and 60 minute intervals of disinfection (Pvalue $>0.05$ ).

This proper effect of silver nanoparticles on $S$. aureus has studied in some other research. A 100\% reduction of S. aureus was indicated following 48 hours contact time of silver nanoparticle-coated masks in Li study (16). Plastic catheters coated with silver nanoparticles were contaminated with S. aureus and 100\% effect of eradicating was observed following 72 hours in David Roe and colleagues study (28). 12.5ppm silver nanoparticles in a culture media showed a $100 \%$ reduction of $S$. aureus colony 
count following 5 minutes in Ghazvini and colleagues' study, but the lower dilutions were not efficient. The effective dilution of Ghazvini's study was lower than in the present study. This difference can be explained by the fact that Ghazvini has set silver nanoparticles directly in contact with $S$. aureus in a culture media (29).

Antimicrobial effect of silver nanoparticles on the growth of staphylococcus aureus was reported to be very insignificant in Kim's study. Kim assessed the antimicrobial effect of silver nanoparticles in a culture media, not as a disinfectant of surfaces. They studied low dilutions of this substance with the unit of nanomole. MIC more than 33nM of silver nanoparticles for S. aureus and MIC more than 6.6nM for Escherichia coli were measured (Every 1000 nanomole equal to $1 \mathrm{ppm}$ ). These items may justify the differences between Kim's study and ours (30). We had a zero $P$. aeruginosa colony count following15 minutes of disinfection with silver nanoparticles and more than $99 \%$ reduction after 5 minutes. It means that three dilutions of silver nanoparticles have similar effects on $P$. aeruginosa colony counts of hospital surfaces after a specific disinfection time ( $P$ value $>0.05$ ).

There are other studies about the disinfection efficacy of silver nanoparticles on P. aeruginosa which had various results. Our study has similar findings to Lara and colleagues' research in Mexico which was about bactericidal efficacy of silver nanoparticles on P. aeruginosa (31). 100 ppm silver nanoparticles indicated a $100 \%$ reduction of $P$. aeruginosa colony count following 15 minutes in culture media in Ghazvini's study which was quite similar to ours (29).

David Roe and colleagues' study represented 70\% efficacy after 72 hours contact time. They had experimented on plastic catheters coated with silver nanoparticles contaminated with P. aeruginosa. Their result was not similar to ours; as well, it was unlike other mentioned studies. Considering the different method of this study from other mentioned studies, this difference could be explainable (28). B. cereus' growth reduction was $100 \%$ after 60 minutes disinfection with 300ppm silver nanoparticles as is observed in the results. Actually, growth reduction of other dilutions (100ppm and 200ppm) was rather high (more than 99\%) in 60 minutes. However none of them could bring $B$. cereus colony count to zero. Although the 300ppm silver nanoparticles' efficacy was much more than the others, this difference was not significant ( $P$ value $>0.05$ ).

Ghazvini's research also studied the effectiveness of silver nanoparticles on $B$. cereus. The maximum dilution applied in that study was 200ppmwhich could not bring $B$. cereus colony count to zero in five, 15, 30 minutes and 24 hours. Although this dilution could indicate more than $5 \log$ reduction which was $99 \%$ of the mentioned bacteria colony count. Considering the fact that we gained almost similar results of this dilution in our study and 300ppm could reduce $100 \%$ of colony count following 60 minutes seems to indicate that both studies demonstrated similar efficacy of silver nanoparticles (29).

As revealed, there was a good effect with no remarkable difference in comparing different dilutions of silver nanoparticles on all microorganisms which were applied for contaminated surfaces in a specific time (Figure 1). This proper effect of silver nanoparticles could be highly suitable to decrease all kinds of organisms, considering the fact that hospital surfaces are often infected with multiple organisms. 300ppm silver nanoparticles could bring the number of all bacteria to zero after 60minutes disinfection. This number was near zero after 30minutes. Regarding the fact that we had sporulative Bacillus in this study, it would seem that 300ppm silver nanoparticles could be recommended as a high level disinfectant. Statistical analysis indicated the efficacy of silver nanoparticles disinfection on decreasing colony count of S. aureus and P. aeruginosa has a remarkable difference with decreasing of $B$. cereus colony count. This difference is observed in all studied dilutions but statistical comparison was not possible, because of having low number of samples and zero number of colony counts in at specific time intervals. Considering the fact that bacillus cereus is sporulative probably the efficacy of different dilutions of silver nanoparticles on non-sporulated Gram-positive and Gram-negative bacteria is greater than sporulated ones, such as bacillus cereus.

In David Roe and colleagues' study on the plastic catheters impregnated with silver nanoparticles, the efficacy of this disinfectant on $S$. aureus after 72 hours contact time was $100 \%$ which was more than $70 \%$ efficacy on $P$. aeruginosa. There were no statistical comparisons (28). Our research results have many similarities to Ghazvini and colleagues' study in Mashhad, Iran. We also found the strong bactericidal effect of silver nanoparticles against Gram-positive bacteria like S. aureus and Gram-negative bacteria like $P$. aeruginosa and lower efficacy of this substance on sporulative bacteria like $B$. cereus compared to the effects on S. aureus and P. aeruginosa (29).

The effect of constant dilution of silver nanoparticles on the reduction of hospital surfaces' organisms (S. aureus, $P$. aeruginosa and B. cereus) is different at various times of disinfection. This positive result of extended time on silver nanoparticles' disinfecting $S$. aureus and $P$. aeruginosa revealed in Ghazvini's study. But, this issue was not verifiable for B. cereus. As was mentioned before, Ghazvini's research did not study surfaces, so there is a definitive difference between that study and ours (29).

The strong efficacy of silver nanoparticles on reducing bacterial organisms' growth (more than 99\%) is the result of this study which mirror similar achievements of prior studies which support the significant viability of silver nanoparticles as a viable alternative for hospital common area disinfectants. Silver nanoparticles of lower dilutions 
(100pm) can also be applied suspect surfaces without any worry of having less effectiveness as a disinfectant. Therefore, there appears to be no need to use a higher concentration of silver nanoparticles which makes them a more affordable alternative.

\section{Acknowledgements}

The authors would like to thank the personnel of infectious diseases ward of Ahvaz Razi Hospital and laboratory of microbiology of Jundishapur University of Medical Sciences for their help and also appreciate the Nano Nasb Pars Company that had provided us silver nanoparticles free of charge for the study.

\section{Financial Disclosure}

None Declared.

\section{Funding Support}

This study was supported by research center of infectious and tropical diseases of Ahvaz Jundishapur University of Medical Sciences, Iran.

\section{Authors' Contribution}

None declared.

\section{References}

1. Peleg Anton Y, Hooper David C. Hospital-Acquired Infections Due to Gram-Negative Bacteria. New Engl J Med. 2010;362(19):1804-1813

2. Kohn WG, Harte JA, Malvitz DM, Collins AS, Cleveland JL, Eklund KJ. Guidelines for infection control in dental health care settings--2003. J Am Dent Assoc. 2004;135(1):33-47

3. Sehulster L, Chinn RY. Guidelines for environmental infection control in health-care facilities. Recommendations of CDC and the Healthcare Infection Control Practices Advisory Committee (HICPAC). MMWR Recomm Rep. 2003;52(RR-10):1-42

4. Weber DJ, Rutala WA, Wenzel RP. Environmental issues and nosocomial infections. In: Weber DJ, Rutala WA, Wenzel RP, editors.Prevention and control of nosocomial infections.Baltimore: Williams and Wilkins; 1997. p. 491-14

5. Bhalla A, Pultz NJ, Gries DM, Ray AJ, Eckstein EC, Aron DC, et al. Acquisition of nosocomial pathogens on hands after contact with environmental surfaces near hospitalized patients. Infect Control Hosp Epidemiol. 2004;25(2):164-7

6. Ray AJ, Hoyen CK, Taub TF, Eckstein EC, Donskey CJ. Nosocomial transmission of vancomycin-resistant enterococci from surfaces. JAMA. 2002;287(11):1400-1

7. Mandell GL, Bennett JE, Dolin R, Rutala W. Principles and practices of infectious diseases. In: Mandell GL, Bennett JE, Dolin R, Rutala W, editors.Disinfection, sterilization, and control of hospital waste.New York: Livingstone; 2010. p. 3677-95

8. Chataigner D, Garnier R, Sans S, Efthymiou ML. [Acute accidental poisoning with hospital disinfectant. 45 cases of which 13 with fatal outcome]. Presse Med.1991;20(16):741-3

9. Weber DJ, Rutala WA, Rutala WA. Occupational risks associated with the use of selected disinfectants and sterilants. In: Weber DJ, Rutala WA, Rutala WA, editors.Disinfection, sterilization, and antisepsis in healthcare.New York: Polyscience Publications; 1998. p. $211-26$

10. Cokendolpher JC, Haukos JF. The Practical Application of Disinfection and Sterilization in Health Care Facilities. Chicago: Ameri- can Hospital Association. 1996

11. Oie S, Kamiya A. Assessment of and intervention for the misuse of aldehyde disinfectants in Japan. Infect Control Hosp Epidemiol. 2002;23(2):98-9

12. Rutala William A, Weber David J. Disinfection and Sterilization in Health Care Facilities: What Clinicians Need to Know. Clin Infect Dis. 2004;39(5):702-709

13. Rotter ML, Wenzel RP . Handwashing, hand disinfection, and skin disinfection. In: Rotter ML, Wenzel RP, editors.Prevention and control of nosocomial infections.Baltimore: Williams and Wilkins;1997.

14. Rutala WA, Mayhall CG. Selection and use of disinfectants in healthcare. In: Rutala WA, Mayhall CG, editors.Infect. Control and Hosp.Philadelphia: Lippincott Williams \& Wilkins; 1999. p. 1161-87

15. Weber DJ, Rutala WA, Block SS. Use of metals as microbicides in preventing infections in healthcare. In: Weber DJ Rutala WA, Block SS, editors.Disinfection, sterilization, and preservation.Philadelphia: Lippincott Williams \& Wilkins; 2001. p. $415-30$

16. Li Y, Leung P, Yau L. Antimicrobial effect of surjical masks coeted with nanoparticles. J Hospital Infect. 2006;62:58-3

17. Brady Michael J, Lisay Catherine M, Yurkovetskiy Alexander V, Sawan Samuel P. Persistent silver disinfectant for the environmental control of pathogenic bacteria. Am J Infec Cont. 2003;31(4):208-214

18. Albrecht MA, Evan CW, Raston CL. Green chemistry and the health implications of nanoparticles. Green Chem. 2006;8:417-32

19. Rai Mahendra, Yadav Alka, Gade Aniket. Silver nanoparticles as a new generation of antimicrobials. Biotechnol Advs. 2009;27(1):7683

20. Schabes-Retchkiman PS, Canizal G, Herrera-Becerra R, Zorrilla C, Liu HB, Ascencio JA. Biosynthesis and characterization of Ti/Ni bimetallic nanoparticles. Optical Materials. 2006;29(1):95-99

21. Gu Hongwei, Ho PL, Tong Edmond, Wang Ling, Xu Bing. Presenting Vancomycin on Nanoparticles to Enhance Antimicrobial Activities. Nano Letters. 2003;3(9):1261-1263

22. Ahmad Z, Pandey R, Sharma S, Khuller GK. Alginate nanoparticles as antituberculosis drug carriers: formulation development, pharmacokinetics and therapeutic potential. Ind J Chest Dis Allied Sci. 2006;48:171-6

23. Gong P, Li H, He X, Wang K, Hu J, Tan W. Preparation and antibacterial activity of Fe3O4@Ag nanoparticles. Nanotechnology. 2007;18:604-11

24. Feng QL, Wu J, Chen GQ, Cui FZ, Kim TN, Kim JO. A mechanistic study of the antibacterial effect of silver ions on Escherichia coli and Staphylococcus aureus. J Biomed Mater. 2000;52((4)):662-8

25. Morones JR, Elechiguerra JL, Camacho A, Ramirez JT. The bactericidal effect of silver nanoparticles. Nanotechnology. 2005;16:2346-53

26. Sondi Ivan, Salopek-Sondi Branka. Silver nanoparticles as antimicrobial agent: a case study on E. coli as a model for Gram-negative bacteria. J Colloid and Interface Sci. 2004;275(1):177-182

27. n Nelson, Marcato Priscyla D, De Souza Gabriel IH, Alves Oswaldo L, Esposito Elisa. Antibacterial Effect of Silver Nanoparticles Produced by Fungal Process on Textile Fabrics and Their Effluent Treatment. J Biomed Nanotechnol. 2007;3(2):203-208

28. Roe David, Karandikar Balu, Bonn-Savage Nathan, Gibbins Bruce, Roullet Jean-Baptiste. Antimicrobial surface functionalization of plastic catheters by silver nanoparticles. J Antimicrob Chemother 2008;61(4):869-876

29. Ghazvini k. Antibacterial activity of malodor neutralizer contain ing silver nanoparticles. Ferdowsi uni Int Biol Sci. 2009;1(1):37-42

30. Kim Jun Sung, Kuk Eunye, Yu Kyeong Nam, Kim Jong-Ho, Park Sung Jin, Lee Hu Jang, et al. Antimicrobial effects of silver nanoparticles. Nanomedicine: Nanotechnology, Biology and Medicine. 2007;3(1):95-101

31. Lara HumbertoH, Ayala-Núñez NildaV, Ixtepan Turrent LilianadelCarmen, Rodríguez Padilla Cristina. Bactericidal effect of silver nanoparticles against multidrug-resistant bacteria. World Microbiol Biotechnol. 2010;26(4):615-621 